Recepción: 09 / 08 / 2016

Aceptación: 16 / 12 / 2016

Publicación: 06 / 03 / 2017

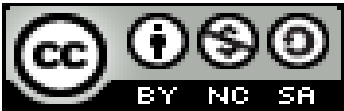

Ciencias de la salud

Artículo de investigación

\title{
Conocimientos sobre salud bucal en los pacientes geriátricos
}

\author{
Knowledge about oral health in geriatric patients
}

\section{Conhecimento sobre saúde bucal em pacientes geriátricos}

Shirley X. Arteaga-Espinoza

shirleyximenaa@gmail.com

Ruth V. Guillén-Mendoza ${ }^{\text {II }}$

ruth-636153@hotmail.com

Juan A. Figueroa-Suárez"II

juanfigueroa_s@gmail.com

Correspondencia: shirleyximenaa@gmail.com

'Magister en Gerencia Educativa, Doctor en Odontología, Docente de la Universidad Laica Eloy Alfaro de Manabí, Manta, Ecuador.

"Magister en Gerencia y Auditoria de Servicios de Salud Bucal, Diploma superior en Odontología Integral, Especialista en Diseño Curricular por Competencias, Doctor en odontología, Docente de la Universidad Laica Eloy Alfaro de Manabí, Manta, Ecuador.

III Magister en Gerencia Educativa, Especialista en Diseño Curricular por Competencias, Analista en Sistemas, Contador Público, Ingeniero en Contabilidad y Auditoría, Docente de la Universidad Laica Eloy Alfaro de Manabí, Manta, Ecuador. 


\title{
Resumen
}

Se realizó un estudio descriptivo y transversal de 80 senescentes, atendidos en la Clínica, en consulta privada desde enero de 2014 hasta abril de 2015, con vistas a identificar los conocimientos sobre salud bucal. La información se recogió en un formulario confeccionado al efecto. Se analizaron las siguientes variables: edad, sexo, conocimiento sobre las funciones principales que tienen los dientes, los efectos de los hábitos nocivos sobre los dientes y la mucosa bucal, cepillado dental, forma y frecuencia de realización. Se utilizaron métodos del nivel teórico y empírico. Se concluyó que la mayoría de los ancianos presentaban conocimientos acerca de las funciones de los dientes, por el contrario existió una repercusión negativa para la salud bucal, el alcoholismo, el tabaquismo y la higiene bucal. Se recomendó la realización de una intervención educativa sobre la importancia de la salud bucal, por los beneficios que les reportara en el orden personal y la importante información que le proporcionara para el diagnóstico de salud odontológico en general.

Palabras clave: anciano; salud bucal; factores de riesgo.

\begin{abstract}
A descriptive and cross - sectional study of 80 senescent patients attended at the Clinic, private consultation from January 2014 to April 2015, was carried out to identify knowledge about oral health. The information was collected in a form prepared for this purpose. The following variables were analyzed: age, sex, knowledge about the main functions of teeth, effects of harmful habits on teeth and oral mucosa, dental brushing, shape and frequency of performance. Methods of the theoretical and empirical level were used. It was concluded that the majority of the elderly presented knowledge about the functioning of the teeth, on the contrary there was a negative repercussion for oral health alcoholism, smoking and oral hygiene. It was recommended that an educational intervention be carried out on the importance of oral health, the benefits that they would receive in the personal order, and the important information they would provide for the diagnosis of dental health in general.
\end{abstract}

Keywords: elderly; oral health; risk factors. 


\section{Resumo}

Um estudo transversal descritivo de 80 senescente, tratado na clínica, prática privada de janeiro 2014 e Abril de 2015, com o objectivo de identificar o conhecimento sobre a saúde oral foi realizada. A informação foi recolhida de uma forma preparada para esse efeito. idade, sexo, conhecimento das principais funções que têm dentes, os efeitos de hábitos nocivos sobre os dentes e mucosa oral, escovação, forma e periodicidade de desempenho: foram analisadas as seguintes variáveis. Foram utilizados métodos de nível teórico e empírica. Concluiu-se que a maioria dos anciãos tinha conhecimento sobre a função dos dentes, por outro lado, houve um impacto negativo sobre a saúde oral, alcoolismo, tabagismo e higiene oral. a realização de uma intervenção educativa sobre a importância da saúde oral, os benefícios para eles em ordem pessoal e irá fornecer informações importantes para o diagnóstico saúde bucal geralmente recomendado.

Palavras chave: idosos; saúde bucal; fatores de risco.

\section{Introducción}

El envejecimiento individual no es un fenómeno exclusivo de la modernidad, puesto que ha estado presente en todas las etapas del desarrollo social y resulta de gran interés para diversas disciplinas, tales como filosofía, arte y medicina; sin embargo, en el siglo XXI ha aumentado progresivamente el número de seres humanos que sobrepasan las barreras cronológicas consideradas por el hombre como ancianidad. Sobre la base de lo anterior, los fines principales de cada país deben estar encaminados a incrementar el nivel de salud de la población, teniendo en cuenta la ética como uno de los elementos fundamentales en el terreno de la salud; por tanto, se impone prestar mayor atención a las demandas particulares de los gerontes y a los problemas que muchos de ellos enfrentan en su contexto diario, sobre todo de índole sanitaria. ${ }^{1}$

Estudios realizados por la OMS sitúa al Ecuador sobre el umbral de los 75 años y, la esperanza de vida es de 76,2 años de edad. ${ }^{2}$

Los primeros signos de envejecimiento del aparato digestivo se observan en la cavidad bucal, como es el cierto grado de oscurecimiento y desgaste de los dientes, xerostomía y atrofias de la 
mucosa bucal, etcétera. En cambio otros, son resultado de la acción de enfermedades o de medicamentos y de efectos de factores socioculturales y económicos. ${ }^{3,4}$

El estado de la salud bucal en el adulto mayor, dependerá del resultado de los hábitos de higiene y cuidado bucal en el hogar de residencia de la persona, sea en el seno de su familia o en una institución geriátrica. Dependerá, también del grado de incapacidad física, de la presencia de enfermedades sistémicas asociadas, de la posibilidad de acceso a los servicios de salud y de las concepciones y valoración que se tenga de la salud bucal. ${ }^{5}$

El denominado grupo de la tercera edad, actualmente ocupa un espacio cada vez más importante en la sociedad, aunque acuden poco a los servicios odontológicos, sí lo hace en general, a servicios sanitarios. Dentro de las causas de esta diferencia se enumeran varias como:

- El difícil acceso a los servicios bucodentales,

- El bajo nivel socioeconómico mayoritario,

- El miedo a ser tratados

- La baja motivación respecto a la salud oral. ${ }^{5}$

La importancia numérica de este colectivo cobra importancia si se tiene en cuenta el mal estado de la salud bucodental y las altas necesidades de tratamiento que se registra en los datos de estudios epidemiológicos realizados en nuestro entorno. ${ }^{5}$

La realización no adecuada de la prevención y de los tratamientos dentales han provocado que un alto porcentaje de adultos mayores presentan pérdida parcial o total de piezas dentarias, lo cual trae como consecuencias que no pueda masticar bien; no puede relacionarse en forma cómoda porque su dicción y fonación están alteradas; la apariencia estética es deficiente, todo lo cual le impide mantener una relación social normal, tendiendo a caer en el aislamiento. ${ }^{6}$

Los profesionales que se ocupan de atender a los ancianos, deben hacerlo desde un ángulo positivo y realista. Aquellos que no creen que las personas de edad son capaces de mantener una calidad de vida aceptable, no se esforzarán por asistirles convenientemente para que logren alcanzarla. 


\section{Material y método}

Se realizó un estudio descriptivo transversal en consulta privada, con el objetivo de identificar los conocimientos de los geriátricos acerca de la salud bucal.

El universo de trabajo estuvo formado por los 80 gerontes que se encontraban en la institución cuando se realizó el examen bucal, donde cada anciano constituyó la unidad de análisis y que dieron su consentimiento por escrito de participar en la misma, a los que se les explicó el propósito de la investigación y los beneficios esperados.

Las variables estudiadas fueron: edad, sexo, conocimiento sobre las funciones principales que tienen los dientes, los efectos de los hábitos nocivos sobre los dientes y la mucosa bucal, cepillado dental, forma y frecuencia de realización, considerando de correctos, aquellos que se cepillan sus dientes y prótesis tres o más veces al día. Los momentos de realización correctos son: al levantarse, después de cada comida y al acostarse y la forma de realización con movimientos de la encía al diente.

La información se obtuvo, mediante la aplicación del cuestionario a los senescentes por parte del autor y colaboradores calibrados con anterioridad, donde se le explicaron los objetivos del estudio, solicitando de ellos la mayor sinceridad y cooperación con las respuestas.

\section{Resultado y discusión}

Tabla 1 Distribución de adulto mayor de acuerdo a la edad y sexo.

\begin{tabular}{|l|c|c|c|c|c|c|}
\hline \multirow{2}{*}{ Edad } & \multicolumn{2}{|l|}{ Masculino } & \multicolumn{2}{l|}{ Femenino } & Total \\
\cline { 2 - 7 } & No & $\%$ & No & \% & No & \\
\hline $60-69$ & 26 & 74.2 & 27 & 60 & 53 & 66.2 \\
\hline $70-79$ & 6 & 17.1 & 10 & 22.2 & 16 & 20 \\
\hline+80 & 3 & 8.5 & 8 & 17.7 & 11 & 13.7 \\
\hline Total & $\mathbf{3 5}$ & $\mathbf{4 3 . 7}$ & $\mathbf{4 5}$ & $\mathbf{5 6 . 2}$ & $\mathbf{8 0}$ & $\mathbf{1 0 0}$ \\
\hline
\end{tabular}


En la composición por edad y sexo (tabla 1), se evidencia el predominio acentuados de las mujeres (56. $2 \%$ ), la cual pudiera ser el efecto de la sobre mortalidad masculina (43.7 \%). Cabe destacar la mayor proporción de personas menores de 79 años, lo cual está acorde con la expectativa de vida del país. Los resultados obtenidos coinciden con la investigación realizada por González Ramos, ${ }^{6}$ siendo el grupo de edad más significativo el de 60 a 69 años, y la distribución según el sexo.

Otro estudio realizado por Blanca Soto $^{7}$ y colaboradores acerca de la modificación de conocimiento sobre salud bucal en adultos mayores institucionalizados, en la casuística primaron los varones $(61.5 \%)$ y el grupo de edades de 75 y más años $(50.0 \%)$, no coincidiendo con los resultados de esta investigación.

Tabla 2 Distribución de los adultos mayores según el conocimiento acerca de las funciones de los dientes.

\begin{tabular}{|c|c|c|}
\hline Funciones & Ancianos & \% \\
\hline Estética & 45 & 56.2 \\
\hline Fonación & 70 & 87.5 \\
\hline Masticación & 71 & 88.7 \\
\hline $\begin{array}{c}\text { Expresión } \\
\text { facial }\end{array}$ & 49 & 61.2 \\
\hline No sabe & 10 & 12.5 \\
\hline
\end{tabular}

Como se muestra en la tabla 2 , del total de pacientes examinados, $88.7 \%$ conocen que la masticación es una de las funciones de los dientes, seguido de la fonación $87.5 \%$, el $61.2 \%$ refieren conocer la función de expresión oral, 88.7 \% . Es de destacar que $12.5 \%$ desconocían las funciones. En investigaciones realizadas se plantea que esto depende, en gran medida del nivel de instrucción que tenga el individuo y la familia, así como la importancia que ésta representa en su escala de valores la salud bucal y la motivación para conservar sus dientes. ${ }^{8,9}$ 
Tabla 3: Distribución de los adultos mayores según el conocimiento acerca de la higiene bucal y las enfermedades bucales.

\begin{tabular}{|c|c|c|}
\hline $\begin{array}{c}\text { Conocimiento sobre } \\
\text { la higiene bucal }\end{array}$ & Ancianos & $\%$ \\
\hline Adecuado & 10 & 12.5 \\
\hline No adecuado & 70 & 87.5 \\
\hline
\end{tabular}

El comportamiento del conocimiento sobre la higiene bucal mostrados en la tabla 3, expresan que solamente el $12.5 \%$ de los ancianos conocen la importancia de la higiene bucal, para evitar la aparición de enfermedades. Después de la sexta década de la vida existe un falso concepto de que es natural el deterior progresivo de las piezas dentarias con el paso del tiempo hasta llegar a la pérdida total, esto hace que los ancianos le quiten importancia a la higiene bucal y su relación con las enfermedades bucales siendo esta la principal herramienta de prevención ante todo tipo de patologías orales como las caries dental y las parodontopatías.

En este orden de ideas, se puede citar que la mala salud oral puede conducir a problemas. Si no se cuidan los dientes y encías, pueden sufrir dolor oral y facial, pérdida de dientes, problemas con el corazón y otros órganos importantes (por las infecciones de boca), problemas de digestión que pueden llevar a una insuficiencia intestinal o a un síndrome de colon irritable, entre otros, ${ }^{10}$ Resultados que coinciden con la investigación realizada por González Ramos. ${ }^{6}$

Tabla 4: Distribución de los adultos mayores según el conocimiento acerca de los hábitos nocivos sobre los dientes y las encías.

\begin{tabular}{|l|l|l|l|l|l|l|}
\hline \multirow{3}{*}{ Conocimiento } & \multicolumn{6}{|c|}{ Hábitos nocivos } \\
\cline { 2 - 7 } & \multicolumn{2}{|c|}{ Tabaquismo } & \multicolumn{2}{c|}{ Alcoholismo } & \multicolumn{2}{c|}{ Bruxismo } \\
\cline { 2 - 7 } & Ancianos & $\%$ & Ancianos & $\%$ & Anciano & $\%$ \\
\hline $\mathrm{Si}$ & 35 & 43.7 & 10 & 12.5 & 18 & 22.5 \\
\hline No & 45 & 56.2 & 70 & 87.5 & 62 & 77.5 \\
\hline Total & $\mathbf{8 0}$ & $\mathbf{1 0 0}$ & $\mathbf{8 0}$ & $\mathbf{1 0 0}$ & $\mathbf{8 0}$ & $\mathbf{1 0 0}$ \\
\hline
\end{tabular}


En la tabla 4, se aprecia que el $56.2 \%$ de los ancianos desconocen que el hábito de fumar es perjudicial para la salud bucal, y el $87.1 \%$ desconocen los efectos del alcohol sobre los dientes y mucosas.

Con referencia a lo anterior, el alcohol se asocia con el origen y la evolución de las enfermedades gingivales y periodontales. Reseca la mucosa bucal y la hace más débil, factor que provoca una más alta susceptibilidad a problemas bucales que el resto de individuos que no beben. El alcohol es el principal causante de la atrofia epitelial de la mucosa bucal, aumenta la permeabilidad de la misma e incrementa la solubilidad de las sustancias tóxicas (como aquellas que encontramos en el tabaco), haciéndonos más vulnerables a enfermedades bucodentales. ${ }^{11}$

Cabe decir, que el hábito de fumar constituye un factor de riesgo en la aparición de muchas afecciones, y en este estudio presenta una significación estadística relevante, predominaron las lesiones bucales en los ancianos fumadores.

Con respecto al hábito bruxismo 77. $5 \%$ desconocen lo perjudicial que resulta para la salud. Según estudios recientes ${ }^{12}$, las fuerzas incrementadas en la mordida, como en el bruxismo, estarían relacionadas con una disminución considerable del aumento de noradrenalina inducido por el estrés en el cerebro, así como con la formación de úlcera estomacal; por lo tanto, el bruxismo, según estos estudios, contribuiría a la capacidad del individuo para manejar el estrés.

Tabla 5: Distribución de los adultos mayores según los conocimientos acerca del cepillado dental.

\begin{tabular}{|l|c|c|c|c|}
\hline $\begin{array}{c}\text { Conocimiento sobre el } \\
\text { cepillado }\end{array}$ & \multicolumn{2}{|c|}{ Resp correcta } & \multicolumn{2}{c|}{ Resp incorrecta } \\
\cline { 2 - 5 } & No & $\%$ & No & $\%$ \\
\hline Frecuencia del cepillado & 26 & 32.5 & 54 & 67.5 \\
\hline Momento de realización & 6 & 7.5 & 74 & 92.5 \\
\hline Forma de cepillado & 3 & 3.7 & 77 & 96.5 \\
\hline
\end{tabular}

En la serie prevaleció el desconocimiento con respecto al cepillado dental, la frecuencia del cepillado con respuesta incorrecta $(67.5 \%)$, momento de realización $(92.5 \%)$ y $96.5 \%$ a la forma 
del cepillado también fue calificada de incorrecta (tabla 5). Resultó inadecuado sobre cuidados, uso y mantenimiento de prótesis y puentes removibles.

Varios de las investigaciones revisadas plantean que la limpieza diaria y frecuente es esencial para remover la placa y mantener una boca saludable, por lo que el cepillado dental contribuye a minimizar los factores de riesgo de caries y enfermedad periodontal, y por consiguiente a disminuir la incidencia de estas enfermedades. ${ }^{13,14,15}$

En el estudio realizado por Rodríguez Salva, ${ }^{16}$ se observa un predominio de la frecuencia incorrecta del cepillado dental, coincidiendo con el nuestro.

Los estudios de Lázaro Soto, ${ }^{7}$ exponen como resultado que los ancianos cepillan sus dientes tres veces al día, mientras que el estudio de Calleja ${ }^{17}$ muestra un alto predominio de higiene bucal deficiente.

Se concluyó que la mayoría de los ancianos presentaban conocimientos acerca de las funciones de los dientes, por el contrario existió una repercusión negativa para la salud bucal: el alcoholismo, el tabaquismo y la higiene bucal. Se recomendó la realización de una intervención educativa sobre la importancia de la salud bucal, por los beneficios que les reportará en el orden personal y la importante información que le proporcionara para el diagnóstico de salud odontológico en general.

\section{Referencias bibliográficas}

1- Corona Carpio M H, Duharte Escalante A, Navarro Nápoles J, Ramón Jiménez R, Díaz del Mazo L. Factores locales y su relación con lesiones bucales en ancianos portadores de prótesis totales. Rev Medisan. 2017. [citado 2 febrero 2016]; 21(4). Disponible en: http://www.medisan.sld.cu/index.php/san/article/view/1064/html

2- El Comercio. 2016. [citado 2 febrero 2016]. Disponible en: http://www.elcomercio.com/actualidad/ecuador-esperanza-vida-salud-latinoamerica.html

3- Sáez Carriera R, Carmona M, Jiménez Quintana Z. Cambios bucales en el adulto mayor. Rev Cubana Estomatol. 2007 [citado 2 de febrero 2016]; 44(4). Disponible en: http://bvs.sld.cu/revistas/est/vol44_4_07/est11407.htm 
4 Alonso P, Sansó FJ, Díaz-Canel AM, Carrasco M, Oliva T. Envejecimiento poblacional y fragilidad en el adulto mayor. Rev Cubana Salud Pública. 2007 [citada 3 de julio 2016]; 33(1). Disponible en: http://bvs.sld.cu/revistas/spu/vol33_1_07/spu10107.htm

5- Meneses Gómez E J. Salud bucodental y calidad de vida Oral en los mayores. 2010 [citada 3 de enero 2016]. Disponible en: eprints.ucm.es/12303/1/T32521.pdf

6- González Ramos R M, Madrazo Ordaz D E, Osorio Núñez M. Conocimientos sobre salud bucal en los círculos de abuelos. Rev. Cubana de Estomatología 2013 [citado 5 enero 2016]; 50(3). Disponible en: http://www.revestomatologia.sld.cu/index.php/est/article/view/138/32

7- Lázaro Soto B. Frómeta Díaz L, Bychkó Trujillo D, Padró Blanco D y Pérez Capote R. Modificaciones del conocimiento sobre salud bucal en adultos mayores institucionalizados en el municipio de San Luis. MEDISAN 2006 [citado 5 enero 2016]; 10. Disponible en: bvs.sld.cu/revistas/san/vol10_e_06/san02(esp)06.pdf

8- Otero Baxter Y, Lemus Cruz L, Boyle Balbín N, del Valle Zelenenko O. Ponencia. Estado nutricional y adulto mayor portador de prótesis. En: Congreso Internacional Estomatología. Ciudad de la Habana, Cuba; 19- 22 de noviembre de 2007. p. 2-5,10.

9- Ardentey García J, Trujillo Otero PE, Silva Contreras AM, Sixto Iglesias M, Crespo Palacios CL. Estado de salud bucal de la población geriátrica perteneciente a la clínica «Ormani Arenado». Rev. Ciencias Médica 2011 [citado 14 marzo 2016]; 15(2). Disponible en: http://publicaciones.pri.sld.cu/rev-fcm/rev-fcm15-2/Rfv15\%282\%29/060211.html

10- Soto G. La importancia de la higiene bucal. 2011. [citado 3 febrero 2016]. Disponible en: http://www.vix.com/es/imj/salud/2011/10/29/la-importancia-de-la-higiene-bucal

11- Porto N. Alcohol y salud bucal. 2013. [citado 3 febrero 2016]. Disponible en: https://www.propdental.es/blog/odontologia/alcohol-y-salud-bucal/

12- Slavicek R. Sato S., (Bruxism - a function of the masticatory organ to cope with stress. Wien Med Wochenschr, 2004 Dec; 154 (23-24): 584-9. 
13- Hermida M, Santana J C, González YE, Borroto DC. Factores de riesgo del cáncer bucal. MediCiego 2007 [citado 3 febrero 2016]; 13(1):1-10. Disponible en:http://bvs.sld.cu/revistas/mciego/vol13_01_07/articulos/a1_v13_0107.htm

14 Lauzardo García del Prado G, Gutiérrez Hernández M, Quintana Castillo, M, Gutiérrez Hernández N, Fajardo Puig J. Caracterización del estado de salud bucal y nivel de conocimientos en pacientes geriátricos Caricuao. Venezuela Rev haban cienc méd 2009 [citado marzo $2016 \quad 10] ; \quad 8(4)$. Disponible en: http://scielo.sld.cu/scielo.php?script=sci_arttext\&pid=S1729519X2009000400020\&lng=es

15- Rodríguez A, Álvarez L. Repercusiones del envejecimiento de la población cubana en el sector salud. Rev Cubana Salud Pública. 2006 [citado 2016 marzo 19]; 32(2). Disponible en: http://scielo.sld.cu/scielo.php?script=sci_arttext\&pid=S0864 -34662006000200013\&lng=es

16- Rodríguez Salvá A, Alfonso Sagué K, Bonet Gorbea M, Prácticas de salud bucal y características sociodemográfica. Rev Cubana Hig Epidemiol 2008 [citado 2016 marzo 19]; 46(3). Disponible en: http://bvs.sld.cu/revistas/hie/vol46_3_08/hie06308.htm

17- Calleja Martínez YM, González Heredia E, Valle Llagostera G, Castañeda Deroncelé M. Estado de salud bucal en 5 consultorios del municipio Palma Soriano, Santiago de Cuba, 2006. Rev Cubana Estomatol. 2006 [citado 2016 marzo 19]; 43(4). Disponible en: http://bvs.sld.cu/revistas/est/vol43_04_06/est01406.html 